\title{
Maternal Viral Infection in Early Pregnancy and Risk of Congenital Heart Disease in Offspring: A Prospective Cohort Study in Central China
}

\author{
Tingting Wang ${ }^{1,2}$ \\ Qiongxuan $\mathrm{Li}^{2}$ \\ Lizhang Chen $\mathbb{D}^{2,3}$ \\ Bin $\mathrm{Ni}^{1}$ \\ Xiaoqi Sheng' \\ Peng Huang ${ }^{4}$ \\ Senmao Zhang ${ }^{2}$ \\ Jiabi Qin ${ }^{2,3}$
}

'NHC Key Laboratory of Birth Defect for Research and Prevention, Hunan Provincial Maternal and Child Health Care Hospital, Changsha City, People's Republic of China; ${ }^{2}$ Department of Epidemiology and Health Statistics, Xiangya School of Public Health Central South University, Changsha City, People's Republic of China; ${ }^{3}$ Hunan Provincial Key Laboratory of Clinical Epidemiology, Changsha City, People's Republic of China; ${ }^{4}$ Department of Thoracic Cardiac Surgery, Hunan Children's Hospital, Changsha City, People's Republic of China
Correspondence: Jiabi Qin Department of Epidemiology and Health Statistics, Xiangya School of Public Health, Central South University, NO. 238 Shang Ma Yuan Ling Xiang Xiangya Road, Kaifu District, Changsha, Hunan, 410078,

People's Republic of China

Tel/Fax +073I-848054I4

Email qinjiabil23@I63.com
Purpose: To examine the associations of maternal virus infection in early pregnancy with risk of offspring congenital heart disease (CHD) and its seven common subtypes including atrial septal defect, ventricular septal defect, atrioventricular septal defect, patent ductus arteriosus, Tetralogy of Tallot, pulmonary stenosis, and transposition of the great arteries.

Patients and Methods: A prospective cohort study was conducted in Central China. A total of 44,048 pregnant women with singleton pregnancies at 8-14 gestational weeks were finally included and followed to 3 months postpartum. Serum was tested for virus infection including hepatitis $\mathrm{B}$ virus (HBV), coxsackievirus-B, human cytomegalovirus (HCMV), herpes simplex virus (HSV), and rubella virus. Multivariable modified Poisson regression models were used to estimate the relative risks (RRs) of all CHDs as well as seven common subtypes of CHD in offspring of pregnant women with different types of virus infection in early pregnancy, adjusting for confounders identified by directed acyclic graphs. Results: At the end of follow-up, 564 births were diagnosed with CHD. Multivariable analyses showed that the presence of maternal viral infection in early pregnancy was independently associated with increased risks of CHD in offspring, with an adjusted relative risk of 2.21 (95\% CI: 1.66-2.95) for HBV infection, 2.21 (95\% CI: 1.63-3.00) for coxsackievirus-B infection, 3.12 (95\% CI: 2.44-3.98) for HCMV infection, and 2.62 (95\% CI: 1.95-3.51) for rubella virus infection. More specifically, the offspring of pregnant women with HCMV infection had the highest increased risk of patent ductus arteriosus ( $R R=10.50,95 \% \mathrm{CI}: 6.24-17.66)$. These findings persisted in analyses that were further adjusted for the other virus of interest in this study. Conclusion: Our study proposed evidence that maternal virus infection in early pregnancy, including HBV, coxsackievirus-B, HCMV, and rubella virus, was implicated in CHD, although more studies remain needed to verify the results, especially associations in specific CHD phenotypes.

Keywords: congenital heart disease, cohort, virus, early pregnancy

\section{Introduction}

Congenital heart disease (CHD) is the most common congenital disorder diagnosed in newborns, accounting for one-third of congenital anomalies. ${ }^{1}$ Current researches indicate that CHD affects $\sim 8$ per thousand live births worldwide and $\sim 9$ per thousand live births in China. ${ }^{2,3}$ Despite numerous advances in pediatric cardiac diagnosis and medical care, CHD continues to be a leading cause of infant morbidity and mortality. ${ }^{4,5}$ It also significantly affects the health of children and adults and brings heavy burden to families and society. ${ }^{6-9}$ Identification of risk factors for CHD as well as subsequent efforts to prevent CHD remain important priorities for research. 
Epidemiological studies have suggested that less than $20 \%$ of CHD could be explained by chromosome abnormalities and gene defects, whereas the majority should be caused by the interaction of genetic and environmental factors. ${ }^{10}$ Early pregnancy is a critical period for the development of fetal cardiovascular system, especially for the second to eighth weeks of pregnancy which is the vulnerable window for CHD. ${ }^{11}$ From a prevention perspective, it is crucial to explore and identify risk factors related to CHD, especially those that can be intervened.

Since the first report on the devastating teratogenic effects of the rubella virus on fetal cardiovascular system published in 1941, ${ }^{12}$ a number of studies have assessed the association of maternal rubella virus infection during pregnancy and risk of CHD. ${ }^{13-15}$ Other virus, including hepatitis B virus (HBV), coxsackievirus-B, human cytomegalovirus (HCMV), and herpes simplex virus (HSV) which are common in pregnant women, are also assessed by few studies. To date, the association between maternal rubella virus infection and $\mathrm{CHD}$ has been well documented, ${ }^{13,16}$ yet analyses of other virus in the risk of CHD have yielded inconsistent findings. ${ }^{17-25}$ Given that the exact pathophysiological mechanisms of most CHD remain unknown nowadays, it would be of value to demonstrate whether relationship between maternal virus infection and CHD exist and whether the relationship could further be a potential marker for clinical prevention. However, data from these studies are difficult to assess due to the lack of serological evidence for microbial agents, ${ }^{17,20,26}$ uncertain time of infection during pregnancy, ${ }^{19,23-25}$ and restricted number of CHD. ${ }^{19,21}$ Furthermore, none of these studies have assessed risk for specific CHD phenotypes by maternal virus infection. If the role of maternal virus infection varies in different phenotypes of CHD, then it is possible that the different CHD phenotype constitution may, at least partially, explain these inconsistent findings.

To this end, in the present study, we aimed to estimate the relative risk of total CHD as well as specific CHD phenotypes in the offspring of mothers who were effected with virus during pregnancy through a prospective cohort study. The virus included rubella virus, HBV, coxsackievirus-B, HCMV, and HSV. Given that the key development phase of CHD is the second to eighth weeks during pregnancy, we restricted the time of infection to early pregnancy.

\section{Materials and Methods}

\section{Ethics Statement}

This study was performed in line with the principles of the Declaration of Helsinki. Approval was granted by the Ethics Committee of Xiangya School of Public Health Central South University, and written informed consent was obtained from all mothers. Additionally, it has been registered in Chinese Clinical Trial Registry Center (registration number: ChiCTR1800016635). Each participant was informed of the study protocol and provided written informed consent before completing an intervieweradministered questionnaire as well as providing biological samples (eg, blood sample).

\section{Study Design and Subjects}

This study was nested on a prospective cohort study conducted among pregnant women and their children in the Hunan Provincial Maternal and Child Health Care Hospital, the provincial health center for women and children in Hunan Province, Central China. This hospital was founded in 1947 and is one of the oldest Maternal and Child Health Hospitals in China. From March 13, 2013 to December 31, 2019, pregnant women who received their first antenatal care between 8 and 14 weeks of gestation were approached and invited to join the cohort. Gestational weeks were estimated using the last menstrual period data or calculated by ultrasound if the menstruation was irregular. ${ }^{27}$ After recruitment, participants who provided informed consent were interviewed face-to-face by trained investigators using a structured questionnaire to collect information on socio-demographic characteristics, obstetric, clinical, and genetic characteristics, and healthrelated factors. All participants were followed up until 3 months after birth to collect detailed information on infant illness, especially birth defects. Data on disease diagnosis were confirmed by medical records.

Pregnant women over 18 years or older who intended to receive prenatal care and to deliver at the study hospital were invited to participate in the cohort. After written informed consents, a total of 49,158 eligible pregnant women were recruited in the present study during their first prenatal care in the early stage of pregnancy. After excluding participants with multiple pregnancies, with termination of pregnancy by artificial abortion or induced labor, still pregnant at the end of follow-up or lost to follow-up, as well as those without data on the status of virus infection in early pregnancy and those whose children were diagnosed with a chromosomal 
aberration or syndromic CHD, a total of 44,048 pregnant women were included in the final analysis (Figure 1).

\section{Specimen Collection and Laboratory Testing}

A 3-5mL peripheral venous blood sample was obtained from each participant under strict hygiene and safety guidelines. Serum separated from blood samples was tested for virus infection including HBV, coxsackievirusB, HCMV, HSV, and rubella virus, at the Hunan Provincial Maternal and Child Health Care Hospital laboratory. The method of enzyme-linked immunoassay (ELISA) was used to detect antibody to hepatitis B surface antigen (HBsAb), hepatitis B surface antigen (HBsAg), antibody to hepatitis $\mathrm{B}$ core antigen ( $\mathrm{HBcAb})$, hepatitis $\mathrm{B}$ e-antigen (HBeAg), and antibody to hepatitis $\mathrm{B}$ e-antigen ( $\mathrm{HBe} A b)$, using commercial kits manufactured by the Beijing Wantai Biological Pharmacy Enterprise Co. Ltd. (Beijing, China). HBV-DNAs was tested by using polymerase chain reaction (PCR) fluorescence probing technology reagents produced by Hunan Sansure Biotech Inc. For the purpose of our study, samples with either HBsAg and HBeAg double positive or HBsAg positive with HBVDNAs level $>2 \times 105 \mathrm{IU} / \mathrm{mL}$ were considered HBV positive. HCMV-specific IgG and IgM were detected by using a commercial chemiluminescence microparticle immunoassay (CMIA) (Diasorin, Italy) based on the manufacturer's instructions; according to manufacturer's recommendations, HCMV-specific IgM values $>22.0 \mathrm{AU} /$ $\mathrm{mL}$ and $\mathrm{HCMV}$-specific $\mathrm{IgG}$ values $>14.0 \mathrm{AU} / \mathrm{mL}$ were

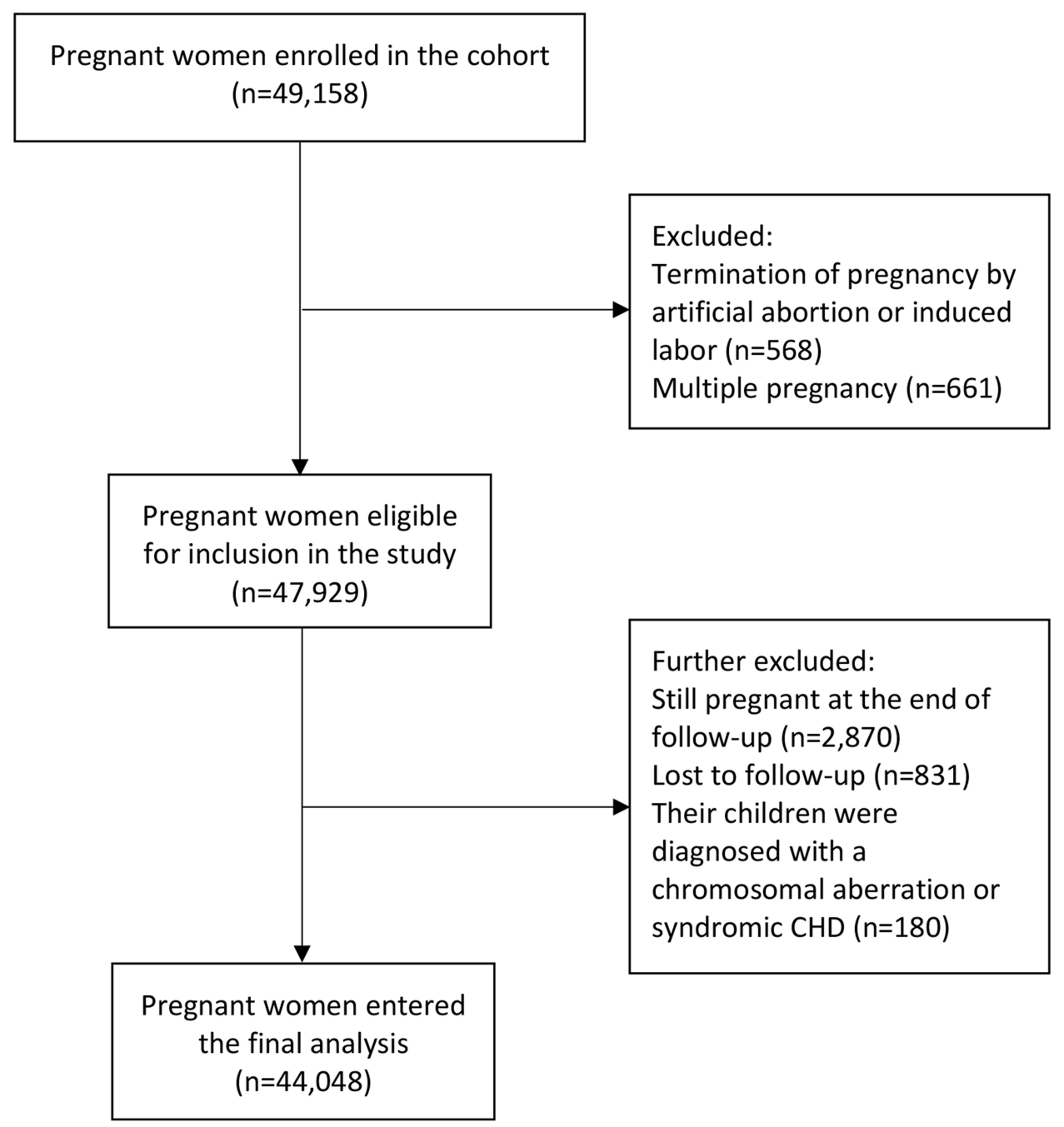

Figure I Flow chart illustrating the process of participants recruitment Abbreviation: $\mathrm{CHD}$, congenital heart disease. 
considered as positive. Detection of HSV infection in the blood samples was done by CMIA kit (Diasorin, Italy); seropositivity was defined as HSV specific IgM values $>2.0 \mathrm{AU} / \mathrm{mL}$ and $\mathrm{HSV}$ specific IgG values $>1.1 \mathrm{AU} / \mathrm{mL}$ as referred to product protocol. Also, CMIA kit (Diasorin, Italy) was used to detect serum rubella virus-specific IgG and IgM; according to the kit, $\operatorname{IgM}$ values $>25 \mathrm{AU} / \mathrm{mL}$ and IgG values $>10 \mathrm{AU} / \mathrm{mL}$ were considered as positive. In addition, the coxsackievirus-B IgM in serum samples was measured with the enzyme-linked immunoassay kits manufactured by the Beijing Beier Bioengineering Co., Ltd (Beijing, China); IgM positivity was a marker for coxsackievirus-B infection.

\section{Diagnosis of CHD}

All CHD cases were diagnosed by pediatric cardiologists through physical examination, heart auscultation, electrocardiogram, pulse oximetry, and echocardiography. The classification of CHD was based on the International Classification of Diseases-10 (ICD-10) diagnosis codes (Q20-Q28). CHD phenotypes including atrial septal defect (ASD) (Q21.0), ventricular septal defect (VSD) (Q21.1), atrioventricular septal defect (AVSD) (Q21.2), patent ductus arteriosus (PDA) (Q25.0), Tetralogy of Fallot (TOF) (Q21.3), pulmonary stenosis (PS) (Q25.6), and transposition of the great arteries (TGA) (Q20.3) were assessed in this study.

\section{Covariates}

At baseline, trained investigators collected information on socio-demographic characteristics, obstetric, clinical, and genetic characteristics, and health-related factors. Sociodemographic characteristics included age, ethnicity, and educational level. Age was divided into four groups: $<25$, 25-29.9, 30-34.9, and $\geq 35$ years. Ethnicity was classified into Han and minority (the other 55 ethnicities in Hunan Province except Han). Educational level was classified as junior high school or below, senior middle school, college, and master or above. Obstetric, clinical, and genetic characteristics included mode of conception, consanguineous marriage (ie, yes or no), parity, history of adverse pregnancy outcomes (ie, yes or no), and family history of congenital malformations (ie, yes or no). Mode of conception was classified into two categories: spontaneous conception and assisted conception. Parity was categorized as primiparous and multiparous. Health-related factors included prepregnancy body mass index (BMI), pre-pregnancy diabetes mellitus (ie, yes or no), personal history of congenital malformations (ie, yes or no), taking folic acid in 3 months before pregnancy or in early pregnancy (ie, yes or no), smoking in early pregnancy (ie, yes or no), drinking in early pregnancy (ie, yes or no), environmental pollution around the dwelling place in 3 months before pregnancy or in early pregnancy (ie, yes or no), and exposure to radioactive hazardous while at work in 3 months before pregnancy or in early pregnancy (ie, yes or no). BMI was calculated as the self-reported body weight in kilograms divided by the self-reported body height in meters squared. Pre-pregnancy body mass index was classified into four groups: $<18.5 \mathrm{~kg} / \mathrm{m}^{2}, 18.5-23.9 \mathrm{~kg} / \mathrm{m}^{2}, 24-27.9 \mathrm{~kg} / \mathrm{m}^{2}$, and $\geq 28 \mathrm{~kg} / \mathrm{m}^{2}$.

\section{Statistical Analysis}

This study was reported following the STrengthening the Reporting of OBservational studies in Epidemiology (STROBE) statement. To avoid data entry errors such as out-of-range values and mismatches, all data were entered using a double-check strategy to guarantee data accuracy in EpiData version 3.1 (EpiData Association, Odense, Denmark). The directed acyclic graph (DAG), a type of causal diagram, was used to identify variables that could confound the association between maternal virus infection and offspring risks of CHD. The DAG has been considered as an alternative to traditional epidemiological methods for confounder identification since it explicitly shows and facilitates the process of causal-inference. ${ }^{28}$ Specifically, compared with data-driven methods for model building, the DAG concentrate on theoretical causal relations between variables when identifying potential confounding factors. ${ }^{29}$ This approach has been used in epidemiology to identify sets of confounding factors (minimal sufficient adjustment set) that can control for potential confounding between exposures and outcomes under the hypothesized causal relationships. In the present study, the DAGitty online software ${ }^{30}$ was used to create DAGs and identify the minimal sufficient adjustment sets for the association between maternal virus infection and offspring risks of CHD (Online Resource Supplementary

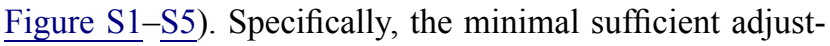
ment sets included educational level, age, ethnicity, history of adverse pregnancy outcomes, pre-pregnancy BMI, and pre-pregnancy diabetes mellitus for HBV infection; educational level, age, and ethnicity for coxsackievirus-B infection; educational level, age, ethnicity, parity, and history of adverse pregnancy outcomes for HCMV infection; educational level, age, ethnicity, parity, and history of adverse 
pregnancy outcomes for HSV infection; educational level, age, ethnicity, parity, history of adverse pregnancy outcomes, and pre-pregnancy BMI for rubella virus infection.

Descriptive statistics were used to assess the characteristics of study participants, and Chi-square test was employed to compare categorical variables. Modified Poisson regression with robust error variances was used in three models to estimate risk ratios (RRs) and their corresponding 95\% confidence intervals (CIs) for CHD in offspring exposed to maternal viral infection in early pregnancy. In the crude model (model 1), RRs were not adjusted for any variable. Model 2 adjusted for variables indicated in the DAGs. Model 3 adjusted for the variables in model 2 plus the other four types of virus infection except itself among the five types of virus infection analyzed in this study.

Two sensitivity analyses were conducted by reanalyzing the association between viral infection in early pregnancy and offspring risks of CHD after 1) excluding pregnant women whose children had any congenital disease other than CHD and 2) excluding pregnant women whose children had more than one CHD phenotypes. All statistical analyses were conducted by using SPSS version 20.0 (SPSS Inc., Chicago, IL, USA) and a p-value $\leq 0.05$ was considered statistically significant.

\section{Results}

A total of 44,048 eligible pregnant women with singleton pregnancies were included for analysis. Based on the blood test results, 7706 pregnant women were identified with virus infection. Among them, 1076 had infections from more than one type of virus. When classified by the type of virus infected, 2480 pregnant women were positive for HBV, 2084 were positive for coxsackievirus-B, 2472 were positive for HCMV, 586 were positive for $\mathrm{HSV}$, and 1290 were positive for rubella virus. In the offspring of these pregnant women, 564 were diagnosed as having CHD, specifically, 170 as having ASD, 246 as having VSD, 52 as having AVSD, 84 as having PDA, 36 as having TOF, 44 as having PS, and 28 as having TGA. The positive rates for each virus as well as the prevalence rates for each type of CHD are shown in Table 1.

\section{Sample Characteristics}

The distribution of baseline characteristics according to the status of maternal viral infection in early pregnancy is summarized in Table 2. Pregnant women with a positive
Table I Prevalence of Maternal Viral Infection in Early Pregnancy and CHD in Offspring

\begin{tabular}{|l|c|c|}
\hline & Number of Cases & Prevalence (95\% Cl) \\
\hline Infected virus & & \\
HBV & 2480 & $5.63 \%(5.41-5.85 \%)$ \\
Coxsackievirus-B & 2084 & $4.73 \%(4.53-4.93 \%)$ \\
HCMV & 2472 & $5.61 \%(5.40-5.83 \%)$ \\
HSV & 586 & $1.33 \%(1.22-1.44 \%)$ \\
Rubella virus & 1290 & $2.93 \%(2.77-3.09 \%)$ \\
Type of CHD & & \\
Total CHD & 564 & $12.80 \%(11.75-13.85 \%)$ \\
ASD & 170 & $3.86 \%(3.28-4.44 \% \circ)$ \\
VSD & 246 & $5.58 \% \circ(4.89-6.28 \% \circ)$ \\
AVSD & 52 & $1.18 \%(0.86-1.50 \% \circ)$ \\
PDA & 84 & $1.91 \% \circ(1.50-2.31 \% \circ)$ \\
TOF & 36 & $0.82 \% \circ(0.55-1.08 \% \circ)$ \\
PS & 44 & $1.00 \%(0.70-1.29 \%)$ \\
TGA & 28 & $0.64 \%(0.40-0.87 \%)$ \\
\hline
\end{tabular}

Abbreviations: ASD, atrial septal defect; AVSD, atrioventricular septal defect; $\mathrm{CHD}$, congenital heart disease; HBV, hepatitis B virus; HCMV, human cytomegalovirus; HSV, herpes simplex virus; PDA, patent ductus arteriosus; PS, pulmonary stenosis; TGA, transposition of the great arteries; TOF, Tetralogy of Fallot; VSD, ventricular septal defect.

infection status for any of the five viruses listed below are classified into the "positive" group: HBV, coxsackievirus-B, HCMV, HSV, and rubella virus. Both pregnant women with a positive infection status and those with a negative infection status were concentrated in the age group of 25-29.9 years (51.3\% vs $43.4 \%)$ and $30-34.9$ years $(24.3 \%$ vs $33.9 \%)$, and their educational level was mostly senior middle school (50.6\% vs 53.0\%) and college $(33.7 \%$ vs $22.6 \%)$. Han Chinese pregnant women were more likely to have a positive infection status. In addition, multiparas, pregnant women with consanguineous marriage, and pregnant women who had a family history of congenital malformations were more likely to be virus infected. In terms of health-related factors, most pregnant women with different infection status had a normal pre-pregnancy BMI, that was in the range of $18.5-23.9 \mathrm{~kg} / \mathrm{m}^{2}(71.0 \%$ in positive ones vs $65.6 \%$ in negative ones). Pregnant women who had a history of congenital malformations were more likely to have a positive infection status. Additionally, compared with pregnant women with a negative infection status, pregnant women with a positive infection status had higher proportions in the characteristics including smoking in early pregnancy and exposure to radioactive hazardous while at work in 3 months before pregnancy or in early pregnancy, while had relatively lower proportions in the characteristics including taking folic acid in 3 months 
Table 2 The Distribution of Baseline Characteristics According to Status of Viral Infection in Early Pregnancy

\begin{tabular}{|c|c|c|c|}
\hline \multirow[t]{2}{*}{ Baseline Characteristics } & \multicolumn{3}{|c|}{ Infection Status } \\
\hline & $\begin{array}{l}\text { Negative } \\
(n, \%)\end{array}$ & $\begin{array}{l}\text { Positive } \\
(n, \%)\end{array}$ & p-value \\
\hline \multicolumn{4}{|l|}{ Sociodemographic characteristics } \\
\hline Age (years) & & & $<0.001$ \\
\hline$<25$ & $3894(10.7)$ & $1086(14.1)$ & \\
\hline $25-29.9$ & $15,772(43.4)$ & $3952(51.3)$ & \\
\hline $30-34.9$ & $12,318(33.9)$ & $1874(24.3)$ & \\
\hline$\geq 35$ & $4358(12.0)$ & $794(10.3)$ & \\
\hline Ethnicity & & & $<0.001$ \\
\hline Han & $34,296(94.4)$ & $7522(97.6)$ & \\
\hline Minority & $2046(5.6)$ & $184(2.4)$ & \\
\hline Educational level & & & $<0.001$ \\
\hline Junior high school or below & $610(16.8)$ & $790(10.3)$ & \\
\hline Senior middle school & $19,264(53.0)$ & $3900(50.6)$ & \\
\hline College & $8224(22.6)$ & $2596(33.7)$ & \\
\hline Master or above & $2744(7.6)$ & $420(5.5)$ & \\
\hline \multicolumn{4}{|l|}{ Obstetric, clinical, and genetic characteristics } \\
\hline Mode of conception & & & 0.342 \\
\hline Spontaneous conception & $28,106(77.3)$ & $5998(77.8)$ & \\
\hline Assisted conception & $8236(22.7)$ & $1708(22.2)$ & \\
\hline Parity & & & $<0.001$ \\
\hline Nulliparous & $16,862(46.4)$ & $2700(35.0)$ & \\
\hline Multiparous & $19,480(53.6)$ & $5006(65.0)$ & \\
\hline Consanguineous marriage (yes) & $120(0.3)$ & $64(0.8)$ & $<0.001$ \\
\hline History of adverse pregnancy outcomes (yes) & $14,684(40.4)$ & $3074(39.9)$ & 0.403 \\
\hline Family history of congenital malformations (yes) & $42(0.1)$ & $20(0.3)$ & 0.002 \\
\hline \multicolumn{4}{|l|}{ Health-related factors } \\
\hline Pre-pregnancy BMI $\left(\mathrm{kg} / \mathrm{m}^{2}\right)$ & & & $<0.001$ \\
\hline$<18.5$ & $6076(16.7)$ & $1456(18.9)$ & \\
\hline $18.5-23.9$ & $23,856(65.6)$ & $5468(71.0)$ & \\
\hline $24-27.9$ & $5476(15.1)$ & $586(7.6)$ & \\
\hline$\geq 28$ & $934(2.6)$ & $196(2.5)$ & \\
\hline Pre-pregnancy diabetes mellitus (yes) & $284(0.8)$ & $48(0.6)$ & 0.144 \\
\hline Personal history of congenital malformations (yes) & $354(1.0)$ & $112(1.5)$ & $<0.001$ \\
\hline Taking folic acid in 3 months before pregnancy or in early pregnancy (yes) & $34,750(95.6)$ & $7322(95.0)$ & 0.020 \\
\hline Smoking in early pregnancy (yes) & $478(1.3)$ & $126(1.6)$ & 0.028 \\
\hline Drinking in early pregnancy (yes) & $504(1.4)$ & $60(0.8)$ & $<0.001$ \\
\hline Exposure to environmental pollution around the dwelling place in 3 months before pregnancy or in early pregnancy (yes) & $808(2.2)$ & $112(1.5)$ & $<0.001$ \\
\hline Exposure to radioactive hazardous while at work in 3 months before pregnancy or in early pregnancy (yes) & II $44(3.1)$ & $288(3.7)$ & 0.008 \\
\hline
\end{tabular}

Note: Bold font indicates statistical significance.

Abbreviation: BMI, body mass index.

before pregnancy or in early pregnancy, drinking in early pregnancy, and exposure to environmental pollution around the dwelling place in 3 months before pregnancy or in early pregnancy. Furthermore, the distribution of baseline characteristics according to the infection status of different virus in early pregnancy was summarized in Online Resource Supplementary Table S1 and Supplementary Table S2.

\section{Maternal HBV Infection in Early Pregnancy and Risk of CHD in Offspring}

Figure 2 shows the association between maternal HBV infection in early pregnancy and risk of CHD in offspring. After adjusting for potential confounders including educational level, age, ethnicity, history of adverse pregnancy outcomes, pre-pregnancy BMI, and pre-pregnancy diabetes mellitus (in model 2), the risk estimates of both 
total CHD (RR=2.21, 95\% CI: 1.66-2.95) and VSD $(\mathrm{RR}=4.63$, 95\% CI: 3.22-6.67) significantly increased in offspring of $\mathrm{HBV}$-infected pregnant women compared to HBV-uninfected pregnant women. Further adjustment for other virus infection including coxsackievirus-B, HCMV, HSV, and rubella virus also showed increased risks of total CHD (RR $=2.41,95 \%$ CI: $1.80-3.21)$ and VSD $(\mathrm{RR}=5.28$, 95\% CI: 3.66-7.63) in offspring of HBV-infected pregnant women (in model 3).

\section{Maternal Coxsackievirus-B Infection in Early Pregnancy and Risk of CHD in Offspring}

Figure 3 shows the association between maternal coxsackievirus-B infection and risk of CHD in offspring. After adjusting for potential confounders including educational level, age, and ethnicity (in model 2), the presence of coxsackievirus-B infection in early pregnancy was independently associated with increased risks of total CHD ( $\mathrm{RR}=2.21,95 \% \mathrm{CI}$ : $1.63-3.00)$, VSD ( $R R=3.74,95 \%$ CI: 2.48-5.63), and AVSD $(R R=2.95$, $95 \%$ CI: 1.27-6.85) in offspring. After further adjustment for other virus infection including HBV, HCMV, HSV, and rubella virus, significantly increased risks of total CHD $(\mathrm{RR}=1.56,95 \%$ CI: $1.07-2.27)$ and $\mathrm{VSD}(\mathrm{RR}=3.14,95 \%$ CI: 1.86-5.29) was still observed in offspring of pregnant women with coxsackievirus-B infection (in model 3).

\section{Maternal HCMV Infection in Early Pregnancy and Risk of CHD in Offspring} Figure 4 shows the association between maternal HCMV infection and risk of CHD in offspring. After adjusting for potential confounders identified including educational level, age, ethnicity, parity and history of adverse pregnancy outcomes (in model 2), maternal HCMV infection in early pregnancy was associated with increased risks of total CHD $(\mathrm{RR}=3.12,95 \% \mathrm{CI}$ :

\begin{tabular}{|c|c|c|c|c|}
\hline Type of CHD & Negative $(n=41,528)$ & Positive $(n=2,480)$ & Model 1 & \\
\hline Total CHD & $514(12.38 \% \circ)$ & $50(20.16 \%)$ & $1.63(1.22-2.17)$ & $=$ \\
\hline ASD & $166(4.00 \%)$ & $4(1.61 \%)$ & $0.40(0.15-1.09)$ & 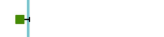 \\
\hline VSD & $214(5.15 \% \circ)$ & $32(12.90 \%$ o $)$ & $2.51(1.73-3.63)$ & 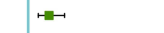 \\
\hline AVSD & $52(1.25 \%)$ & $0(0.00 \%)$ & - & \\
\hline PDA & $78(1.88 \%)$ & $6(2.42 \%)$ & $1.29(0.56-2.95)$ & $n$ \\
\hline TOF & $34(0.82 \%)$ & $2(0.81 \% \circ)$ & $0.99(0.24-4.10)$ & - \\
\hline PS & $42(1.01 \%)$ & $2(0.81 \% \circ)$ & $0.80(0.19-3.30)$ & 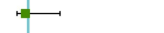 \\
\hline \multirow[t]{2}{*}{ TGA } & $28(0.67 \%)$ & $0(0.00 \%)$ & - & \\
\hline & & & & $\begin{array}{llllll} & 1 & & & & \\
012 & 4 & 6 & 8 & 10 \\
& \text { Risk ratio }\end{array}$ \\
\hline
\end{tabular}
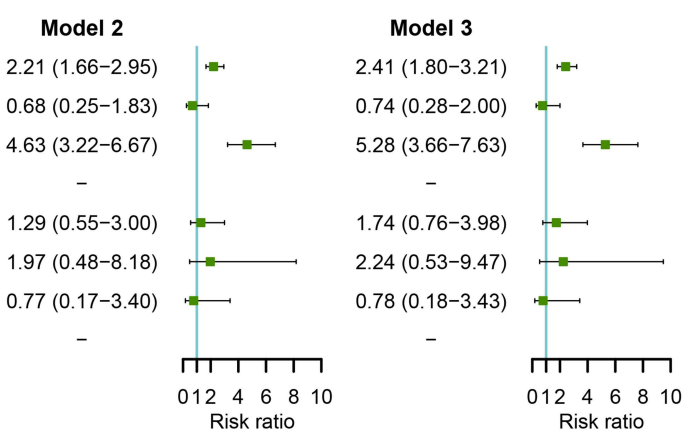

Figure 2 Maternal HBV infection in early pregnancy and risks of CHD in offspring.

Notes: Model I was a crude model without any variable adjusted. Model 2 adjusted for educational level, age, ethnicity, history of adverse pregnancy outcomes, prepregnancy BMI, and pre-pregnancy diabetes mellitus. Model 3 adjusted for educational level, age, ethnicity, history of adverse pregnancy outcomes, pre-pregnancy BMI, prepregnancy diabetes mellitus, and other virus infection including coxsackievirus-B, HCMV, HSV, and rubella virus.

Abbreviations: BMI, body mass index; CHD, congenital heart disease; HBV, hepatitis B virus; HCMV, human cytomegalovirus; HSV, herpes simplex virus.

$\begin{array}{cccc}\text { Type of CHD } & \text { Negative }(\mathbf{n = 4 1 , 9 6 4 )} & \text { Positive }(\mathbf{n = 2 , 0 8 4 )} & \text { Model } \mathbf{1} \\ \text { Total CHD } & 516(12.30 \%) & 48(23.03 \%) & 1.87(1.40-2.51) \\ \text { ASD } & 161(3.84 \%) & 9(4.32 \%) & 1.13(0.58-2.20) \\ \text { VSD } & 219(5.22 \%) & 27(12.96 \%) & 2.48(1.67-3.69) \\ \text { AVSD } & 46(1.10 \%) & 6(2.88 \%) & 2.63(1.12-6.14) \\ \text { PDA } & 80(1.91 \%) & 4(1.92 \%) & 1.01(0.37-2.75) \\ \text { TOF } & 36(0.86 \%) & 0(0.00 \%) & - \\ \text { PS } & 40(0.95 \%) & 4(1.92 \%) & 2.01(0.72-5.62) \\ \text { TGA } & 24(0.57 \%) & 4(1.92 \%) & 3.36(1.17-9.66) \\ & & & \end{array}$
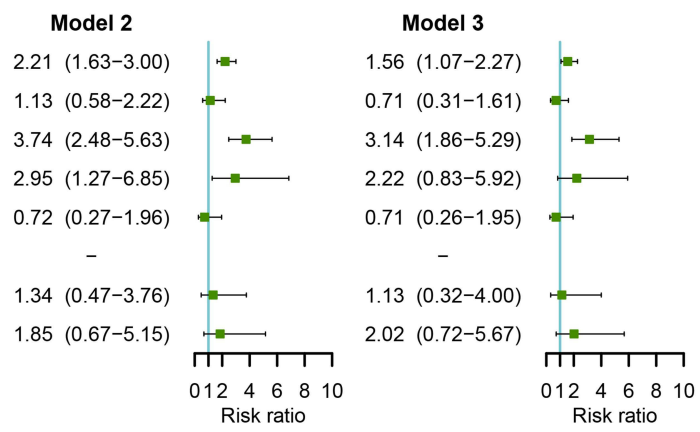

Figure 3 Maternal coxsackievirus-B infection in early pregnancy and risks of CHD in offspring.

Notes: Model I was a crude model without any variable adjusted. Model 2 adjusted for educational level, age, and ethnicity. Model 3 adjusted for educational level, age, ethnicity, and other virus infection including HBV, HCMV, HSV, and rubella virus.

Abbreviations: CHD, congenital heart disease; HBV, hepatitis B virus; HCMV, human cytomegalovirus; HSV, herpes simplex virus. 


$\begin{array}{ccc}\text { Type of CHD } & \text { Negative }(\mathbf{n = 4 1 , 5 7 6}) & \text { Positive }(\mathbf{n = 2 , 4 7 2}) \\ \text { Total CHD } & 482(11.59 \%) & 82(33.17 \%) \\ \text { ASD } & 148(3.56 \%) & 22(8.90 \%) \\ \text { VSD } & 216(5.20 \%) & 30(12.14 \%) \\ \text { AVSD } & 46(1.11 \%) & 6(2.43 \% 0) \\ \text { PDA } & 50(1.20 \% 0) & 34(13.75 \%) \\ \text { TOF } & 30(0.72 \%) & 6(2.43 \% 0) \\ \text { PS } & 38(0.91 \% 0) & 6(2.43 \%) \\ \text { TGA } & 28(0.67 \%) & 0(0.00 \%)\end{array}$
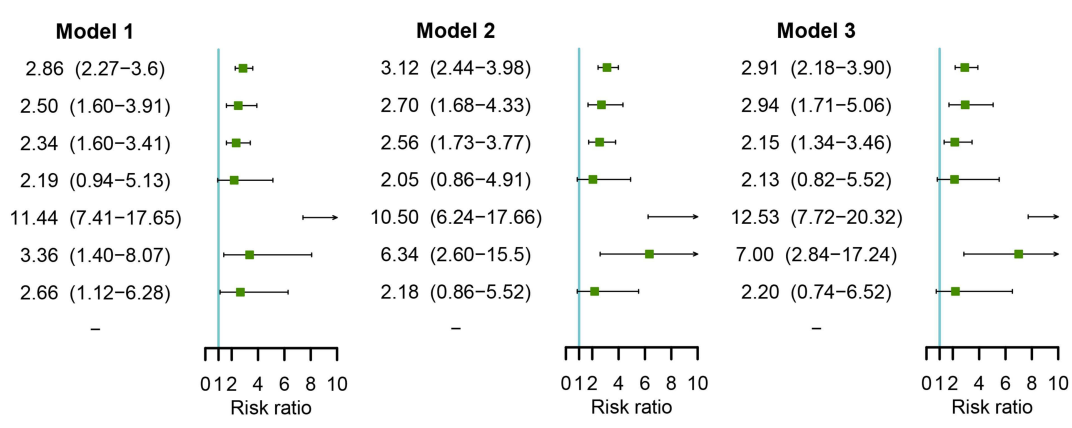

Figure 4 Maternal HCMV infection in early pregnancy and risks of CHD in offspring.

Notes: Model I was a crude model without any variable adjusted. Model 2 adjusted for educational level, age, ethnicity, parity, and history of adverse pregnancy outcomes. Model 3 adjusted for educational level, age, ethnicity, parity, history of adverse pregnancy outcomes, and other virus infection including HBV, coxsackievirus-B, HSV, and rubella virus.

Abbreviations: CHD, congenital heart disease; HBV, hepatitis B virus; HCMV, human cytomegalovirus; HSV, herpes simplex virus.

\begin{tabular}{|c|c|c|}
\hline Type of CHD & Negative $(n=43,462)$ & Positive $(n=586)$ \\
\hline Total CHD & $556(12.79 \% \circ)$ & $8(13.65 \%)$ \\
\hline ASD & $166(3.82 \%)$ & $4(6.83 \%)$ \\
\hline VSD & $241(5.55 \%)$ & $5(8.53 \% \circ)$ \\
\hline AVSD & $50(1.15 \%)$ & $2(3.41 \%)$ \\
\hline PDA & $82(1.89 \%)$ & $2(3.41 \%)$ \\
\hline TOF & $36(0.83 \%)$ & $0(0.00 \%)$ \\
\hline PS & $44(1.01 \% \circ)$ & $0(0.00 \%)$ \\
\hline TGA & $28(0.64 \%)$ & $0(0.00 \%)$ \\
\hline
\end{tabular}
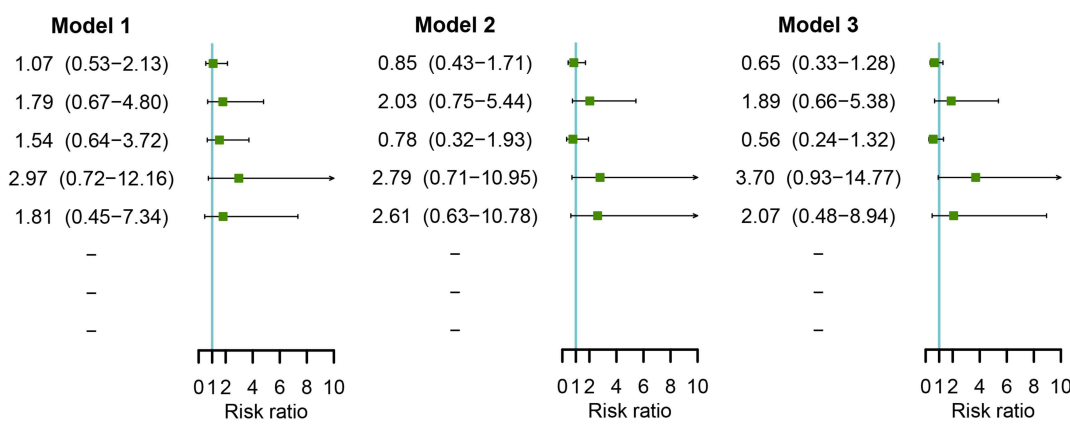

Figure 5 Maternal HSV infection in early pregnancy and risks of CHD in offspring.

Notes: Model I was a crude model without any variable adjusted. Model 2 adjusted for educational level, age, ethnicity, parity, and history of adverse pregnancy outcomes. Model 3 adjusted for educational level, age, ethnicity, parity, history of adverse pregnancy outcomes, and other virus infection including HBV, coxsackievirus-B, HCMV, and rubella virus.

Abbreviations: CHD, congenital heart disease; HBV, hepatitis B virus; HCMV, human cytomegalovirus; HSV, herpes simplex virus.

2.44-3.98) and most CHD subtypes including ASD $(\mathrm{RR}=2.70,95 \% \mathrm{CI}: 1.68-4.33)$, VSD $(\mathrm{RR}=2.56,95 \%$ CI: 1.73-3.77), PDA (RR=10.50, 95\% CI: 6.24-17.66), and TOF $(\mathrm{RR}=6.34,95 \%$ CI: 2.60-15.5). Further adjustment for other virus infection including $\mathrm{HBV}$, coxsackievirus-B, HSV, and rubella virus also showed increased risks of total CHD (RR=2.91, 95\% CI: 2.18 3.90), ASD (RR=2.94, 95\% CI: 1.71-5.06), VSD

$\begin{array}{ccc}\text { Type of CHD } & \text { Negative }(\mathbf{n = 4 2 , 7 5 8}) & \text { Positive }(\mathbf{n = 1 , 2 9 0 )} \\ \text { Total CHD } & 520(12.16 \%) & 44(34.11 \%) \\ \text { ASD } & 153(3.58 \%) & 17(13.18 \%) \\ \text { VSD } & 226(5.29 \%) & 20(15.50 \%) \\ \text { AVSD } & 42(0.98 \% \circ) & 10(7.75 \%) \\ \text { PDA } & 82(1.92 \%) & 2(1.55 \%) \\ \text { TOF } & 34(0.80 \% \circ) & 2(1.55 \%) \\ \text { PS } & 42(0.98 \%) & 2(1.55 \%) \\ \text { TGA } & 24(0.56 \%) & 4(3.10 \%)\end{array}$

Figure 6 Maternal rubella virus infection in early pregnancy and risks of CHD in offspring.

Notes: Model I was a crude model without any variable adjusted. Model 2 adjusted for educational level, age, ethnicity, parity, history of adverse pregnancy outcomes, and pre-pregnancy BMI. Model 3 adjusted for educational level, age, ethnicity, parity, history of adverse pregnancy outcomes, pre-pregnancy BMI, and other virus infection including HBV, coxsackievirus-B, HCMV, and HSV.

Abbreviations: BMI, body mass index; CHD, congenital heart disease; HBV, hepatitis B virus; HCMV, human cytomegalovirus; HSV, herpes simplex virus.]

\section{Model 1}

$2.80(2.07-3.80)$
$3.68(2.24-6.06)$
$2.93(1.86-4.62)$
$7.89(3.97-15.69)$
$0.81(0.20-3.28)$
$1.95(0.47-8.11)$
$1.58(0.38-6.51)$
$5.52(1.92-15.9)$

$$
\text { (1) }
$$

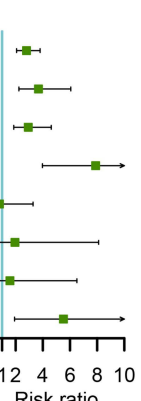

\section{Model 2}

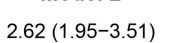

$3.25(2.04-5.19)$

$2.19(1.41-3.41)$

$2.53(1.34-4.80)$

$0.97(0.24-3.91)$

$2.40(0.61-9.43)$

$1.71(0.39-7.46)$

$3.46(1.32-9.05)$

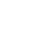


$(\mathrm{RR}=2.15,95 \%$ CI: $1.34-3.46), \mathrm{PDA}(\mathrm{RR}=12.53,95 \%$ CI: 7.72-20.32), and TOF (RR=7.00, 95\% CI: 2.84 17.24) in offspring of HCMV-infected pregnant women (in model 3).

\section{Maternal HSV Infection in Early Pregnancy and Risk of CHD in Offspring}

Figure 5 shows the association between maternal HSV infection and risk of CHD in offspring. No significant association was observed between maternal HSV infection in early pregnancy and risk of total CHD or any CHD subtype in offspring, either by adjusting for potential confounders including educational level, age, ethnicity, parity and history of adverse pregnancy outcomes (in model 2) or by further adjusting for other four types of virus infection including HBV, coxsackievirus-B, HCMV, and rubella virus (in model 3) (all $\mathrm{p}>0.05$ ).

\section{Maternal Rubella Virus Infection in Early Pregnancy and Risk of CHD in Offspring}

Figure 6 shows the association between maternal rubella virus infection and risk of CHD in offspring. After adjusting for potential confounders including educational level, age, ethnicity, parity, history of adverse pregnancy outcomes, and pre-pregnancy BMI (in model 2), maternal rubella virus infection in early pregnancy was associated with increased risks of total $\mathrm{CHD}(\mathrm{RR}=2.62,95 \% \mathrm{CI}$ : 1.95-3.51) and several subtypes of CHD including ASD $(\mathrm{RR}=3.25,95 \% \mathrm{CI}: 2.04-5.19)$, VSD ( $\mathrm{RR}=2.19,95 \% \mathrm{CI}$ : 1.41-3.41), AVSD ( $R R=2.53$, 95\% CI: $1.34-4.80)$, and TGA (RR=3.46, 95\% CI: 1.32-9.05). Further adjustment for other virus infection including HBV, coxsackievirus-B, HCMV, and HSV also showed increased risks of total CHD (RR=2.79, 95\% CI: 2.08-3.73), ASD ( $R R=3.43$, 95\% CI: 2.14-5.49), VSD ( $R R=2.37,95 \%$ CI: 1.53 3.68), AVSD ( $\mathrm{RR}=3.23,95 \% \mathrm{CI}: 1.64-6.39$ ), and TGA $(\mathrm{RR}=3.18,95 \% \mathrm{CI}: 1.21-8.40)$ in offspring of pregnant women infected with rubella virus (in model 3 ).

\section{Sensitivity Analysis}

Sensitivity analyses confirmed previous results. Exclusion of pregnant women whose children had any congenital disease other than CHD ( $\mathrm{n}=1716)$ or exclusion of pregnant women whose children had more than one CHD phenotypes $(\mathrm{n}=109)$ did not materially change the presented results. Data are shown in Online Resource Supplementary Figure S6 and Supplementary Figure S7.

\section{Discussion}

Using a large sample comprising 44,048 subjects, we provided evidence that maternal virus infection in early pregnancy, including HBV, coxsackievirus-B, HCMV, and rubella virus, was independently associated with risk of total CHD and several major CHD phenotypes in offspring. These findings persisted in analyses that were further adjusted for the other virus of interest in this study other than the virus being assessed, and were also confirmed by sensitivity analyses. Given that maternal virus infection in early pregnancy is so common $(5.63 \%$ for $\mathrm{HBV}, 4.73 \%$ for coxsackievirus-B, $5.61 \%$ for $\mathrm{HCMV}$, $1.33 \%$ for HSV, and $2.93 \%$ for rubella virus in the present study population) and CHD are so frequent $(12.80 \%$ in the present study population), even modest teratogenic risks could result in a substantial burden of disease. Our study with better qualification and quantification of these risks can not only provide research insights into teratogenic mechanisms but also be helpful in clinical counseling of infected women and primary prevention.

A number of previous studies were conducted to explore the association between maternal virus infection and the development of CHD. ${ }^{17-26,31-36}$ All studies were case-control by design. In most studies, the diagnosis of maternal virus infection was based on the self-reported data, generally without serologic proof of the infection. ${ }^{17,19,26,31-36}$ Thus, it was difficult for these studies to assess the association of different types of viral infection with CHD in offspring. Only a few studies in which the status of maternal virus infection determined by medical records showed the associations between different types of maternal virus infection in early pregnancy and risk of CHD. ${ }^{8,19,21-25,37}$ Yang et al and Liu et al showed an increased risk of CHD in offspring whose mother were infected with rubella virus or $\mathrm{HCMV}^{24,25}$ whereas Lai et al, Li et al, and Liang et al did not find any increase in CHD after exposure to maternal HBV, HCMV, or HSV infection. ${ }^{18,19,22}$ After adjusting for potential confounders, Guo et al still observed significantly increased risks of CHD in offspring exposed to maternal HBV, HCMV, HSV, rubella virus, and coxsackievirus-B infection in early pregnancy. ${ }^{21}$ However, it is worth noting that all these studies observed the associations in CHD as a whole, without considering possible differences among different subtypes of CHD. In our cohort study, we not only assessed the associations of different types of virus infection in pregnant women during early pregnancy with risk of total CHD, but also analyzed the associations between the exposures and several major CHD phenotypes including ASD, VSD, AVSD, PDA, TOF, PS, and TGA. The results 
showed significantly increased risks of total CHD and at least one phenotype of CHD in offspring whose mothers were infected with coxsackievirus-B, HBV, HCMV, and rubella virus in early pregnancy; however, the size of observed associations varied with types of infected virus as well as CHD phenotypes. From a research and clinical perspective, the results highlight how overall risk estimates for CHD as a group can be uninformative and potentially misleading. The etiologic and development heterogeneity of cardiovascular malformations requires specific assessments by types of cardiovascular malformations. Similarly, exposures, such as virus infection, can be heterogeneous and can benefit from the assessment of the source of infection. A detailed assessment of exposure as well as outcomes not only helps to highlight risk patterns that might be otherwise missed, but also has the potential to provide important information for targeted research and personalized clinical consultation.

The present study has several strengths. It is by far the first study ever to have assessed the association of different types of virus infection in pregnant women during early pregnancy with CHD in the aggregate as well as with specific CHD phenotypes diagnosed by objective methods in such a large and well-defined cohort. The prospective cohort design allowed us to obtain accurate exposure data from standardized laboratory tests and avoided possible recall bias and ensured the reliability of the study. Also, convenient and effective methods of communication between researchers and participants were established, including telephone call, WeChat, and short message, which contributed to reduction in the rate of lost to follow-up (1.7\% in this study). Furthermore, a rich variety of variables related to socio-demographic characteristics, obstetric characteristics, disease and health conditions, history of adverse pregnancy, lifestyle, exposure of environmentally harmful substances, and concurrent infections were evaluated and included as covariates in the statistical analyses.

The study also has several limitations. Firstly, we recruited participants from a single tertiary care center. The results of the study might not reflect those of other institutions across the country. However, the standardized laboratory testing and unified clinical diagnosis within the institution allowed assessment of the effect of maternal virus infection status during early pregnancy on CHD risk without confounding from these variables. Secondly, in China, an autopsy would not be performed on the fetus after selective termination of pregnancy unless requested by the pregnant woman herself. Therefore, even if a pregnant woman chooses to terminate her pregnancy because of the diagnosis of CHDs by fetal echocardiography, it was impossible to obtain accurate data on the proportion of CHDs in pregnancies electively terminated. In such scenario, we were unable to exclude the possibility that the exclusion of elective terminations because of diagnosis of a CHD could bias the results. Thirdly, as with other observational studies, although potential confounders were adjusted as much as possible in the analyses, the possibility of residual confounding caused by unmeasured or unknown covariates could not be ruled out. Fourthly, some findings in the present study could be due to chance; this is a consideration in any epidemiological study, especially in studies such as this that involved multiple comparisons. Fifthly, considering that the key developmental period for fetal heart is second to eighth weeks of pregnancy, the result from evaluation of the association between maternal viral infection and risks of CHD based on the testing for viral infection in this time period are the most convincing. However, it is unlikely to be achieved given the practicalities of the first antenatal care in China. In our study, based on the time of first antenatal care for pregnant women in China, pregnant women who received their first antenatal care between 8 and 14 weeks of gestation were invited to join the cohort. Although the test results might not indicate the active infections in 2-8 gestational weeks, our findings could still provide evidence for the associations of maternal viral infection in early pregnancy and risks of CHD. An additional limitation was the limited sample size of certain exposure and heart defect groups, which reduced the precision of risk estimates and therefore the ability to detect effects when these were present. Risks of specific CHD phenotypes should be investigated further in larger studies.

Our findings of significant associations between maternal virus infection in early pregnancy and risk of CHD suggest the necessity of laboratory tests for coxsackievirus-B, HBV, HCMV, and rubella virus in preparation for pregnancy as well as in early pregnancy, and the importance of timely treatment (if positive). Although our study did not find an association of maternal HSV infection and CHD risk in offspring, testing for HSV is also necessary given the negative effect of HSV infection on morbidity and mortality in pregnant women. Evidence suggests that, given the hormonal changes during pregnancy and resultant effects on the immune system, efforts to decrease the prevalence of virus infection and other diseases by modulating the local or systemic hormonal environment is worthy to be considered. ${ }^{38-40}$ Approaches 
to boost pathogen-specific immunity may provide new pathways for prevention and treatment. Interfering with the specific interactions between certain virus and the placenta may also provide a potential prophylactic and therapeutic strategy. Vaccinations before and during pregnancy have been proved to be safe and effective against some pathogens (such as rubella virus), and could one day be expanded to include vaccines against other pathogens, such as coxsackievirus-B, HCMV, and HSV. ${ }^{38,41}$ Maternal vaccination may bring long-term benefits for the child by reducing fetal and placental inflammation. Overall, the education of women who are in preparation for pregnancy or in pregnancy on prevention of virus infection as well as early detection and appropriate treatment of infectious diseases remain important strategies to protect maternal and infant health and improve the quality of the newborn.

\section{Conclusion}

In conclusion, our study proposed evidence that maternal virus infection in early pregnancy, including coxsackievirus-B, HBV, HCMV, and rubella virus, was implicated in CHD, although more studies remain needed to verify the results, especially associations in specific CHD phenotypes. Also, further studies are needed to clarify underlying biological mechanisms. In the meantime, efforts to prevent virus infection in pregnant women and to treat infected women with appropriate agents may help decrease the prevalence of some subtypes of CHD.

\section{Data Sharing Statement}

The data underlying this article will be shared on reasonable request to the corresponding author.

\section{Ethics Approval and Informed Consent}

This study was performed in line with the principles of the Declaration of Helsinki. Approval was granted by the Ethics Committee of Xiangya School of Public Health Central South University (No. XYGW-2018-36). Informed consent was obtained from all individual participants included in the study.

\section{Consent for Publication}

Additional informed consent was obtained from all individual participants for whom identifying information is included in this article.

\section{Acknowledgments}

The authors would like to thank all colleagues working in Maternal and Child Health Promotion and Birth Defect Prevention Group.

\section{Author Contributions}

TTW and JBQ conceived the study. TTW, QXL, LZC, BN, XQS, PH, and SMZ acquired the data. TTW and QXL performed the data analysis, TTW wrote the first draft. All authors made a significant contribution to the work reported, whether that is in the conception, study design, execution, acquisition of data, analysis and interpretation, or in all these areas; took part in drafting, revising or critically reviewing the article; gave final approval of the version to be published; have agreed on the journal to which the article has been submitted; and agree to be accountable for all aspects of the work.

\section{Funding}

This work was supported by the Project Funded by the National Natural Science Foundation Program of China (82173608, 82073653, 81973137, and 81803313), Hunan Provincial Science and Technology Talent Support Project (2020TJ-N07), and Open Project from NHC Key Laboratory of Birth Defect for Research and Prevention (KF2020006).

\section{Disclosure}

The authors report no conflicts of interest in this work.

\section{References}

1. Mozaffarian D, Benjamin EJ, Go AS, et al. Heart disease and stroke statistics-2016 update a report from the American Heart Association. Circulation. 2016;133:E38-E60. doi:10.1161/CIR.000000000 0000350

2. Liu Y, Chen S, Zuhlke L, et al. Global birth prevalence of congenital heart defects 1970-2017: updated systematic review and meta-analysis of 260 studies. Int J Epidemiol. 2019;48:455-463. doi:10.1093/ije/ dyz009

3. Zhao Q, Ma X, Ge X, et al. Pulse oximetry with clinical assessment to screen for congenital heart disease in neonates in China: a prospective study. Lancet. 2014;384:747-754. doi:10.1016/ S0140-6736(14)60198-7

4. Wren C, Irving CA, Griffiths JA, et al. Mortality in infants with cardiovascular malformations. Eur J Pediatr. 2012;171:281-287. doi:10.1007/s00431-011-1525-3

5. Tennant PWG, Pearce MS, Bythell M, Rankin J. 20-year survival of children born with congenital anomalies: a population-based study. Lancet. 2010;375:649-656. doi:10.1016/S0140-6736(09)61922-X

6. Wang T, Chen L, Yang T, et al. Congenital heart disease and risk of cardiovascular disease: a meta-analysis of cohort studies. J Am Heart Assoc. 2019;8:e012030. doi:10.1161/JAHA.119.012030

7. Gurvitz MZ, Inkelas M, Lee M, Stout K, Escarce J, Chang R. Changes in hospitalization patterns among patients with congenital heart disease during the transition from adolescence to adulthood. $\mathrm{J}$ Am Coll Cardiol. 2007;49:875-882. doi:10.1016/j.jacc.2006.09.051 
8. Marelli AJ, Mackie AS, Ionescu-Ittu R, Rahme E, Pilote L. Congenital heart disease in the general population - changing prevalence and age distribution. Circulation. 2007;115:163-172. doi:10.1161/CIRCULATIONAHA.106.627224

9. Blue GM, Kirk EP, Sholler GF, Harvey RP, Winlaw DS. Congenital heart disease: current knowledge about causes and inheritance. Med J Austr. 2012;197:155-159. doi:10.5694/mja12.10811

10. Pierpont ME, Brueckner M, Chung WK, et al. Genetic basis for congenital heart disease: revisited: a scientific statement from the American Heart Association. Circulation. 2018;138:E653-E711. doi:10.1161/CIR.0000000000000606

11. Gorini F, Chiappa E, Gargani L, Picano E. Potential effects of environmental chemical contamination in congenital heart disease. Pediatr Cardiol. 2014;35:559-568. doi:10.1007/s00246-014-0870-1

12. Gregg NM. Congenital cataract following German measles in the mother. Trans Ophthalmol Soc Aust. 1941;3:35-46.

13. Oster ME, Riehle-Colarusso T, Correa A. An update on cardiovascular malformations in congenital rubella syndrome. Birth Defects Res A. 2010;88:1-8.

14. Jenkins KJ, Correa A, Feinstein JA, et al. Noninherited risk factors and congenital cardiovascular defects: current knowledge a scientific statement from the American Heart Association Council on cardiovascular disease in the young. Circulation. 2007;115:2995-3014. doi:10.1161/CIRCULATIONAHA.106.183216

15. Campbell M. Place of maternal rubella in the aetiology of congenital heart disease. $\mathrm{Br}$ Med J. 1961;1:691-696. doi:10.1136/ bmj.1.5227.691

16. Rosenberg HS. Cardiovascular effects of congenital infections. Am J Cardiovasc Pathol. 1987;1:147-156.

17. Zhang Q, Zhang Y, Lu F, Chen G, Ren D. Epidemiological characteristics and influencing factors of congenital heart disease among school-age children in Qianxinan Prefecture. Chin J Clin Ration Drug Use. 2018;11:98-100.

18. Liang Q, Gong W, Zheng D, Zhong R, Wen Y, Wang X. The influence of maternal exposure history to virus and medicine during pregnancy on congenital heart defects of fetus. Environ Sci Pollut R. 2017;24:5628-5632. doi:10.1007/s11356-016-8198-4

19. Li S, Liu L, Tong Y. Relationship between infection in pregnant women and congenital heart disease in neonates. Chin J Nosocomiol. 2016;26:177-179.

20. Chen X, Wang A, Su H. A case-control study of congenital heart disease risk factors among 1009 infants. Chin J Dis Control Prev. 2016;20:1114-1116.

21. Guo J, Hong X, Yao M. Analysis of the risk factors of congenital heart disease. Chin J Neonatol. 2010;25:76-79.

22. Lai J, Han L, Xu S, Fang Y. A study on the risk factors of neonatal congenital heart disease. J Prev Med Inform. 2001;17:370-371.

23. Liu Z, Tang S, Yang Y, Hu H, Wu T, Pan X. Study on the etiology of congenital heart disease. J Clin Cardiol. 1996;12:25-27.

24. Yang Y, Zhou X, Liu S, Zhao L, Liu Y, Zhang X. A study on the relationship between congenital heart disease and rubella virus and type 1 herpes virus infection. Med J Chin Peoples Liberation Army. 1995;20:273-275.
25. Liu Z, Tang S, Wan C, et al. A study on the relationship between congenital heart disease and cytomegalovirus infection. J Clin Cardiol. 1994;10:287-288.

26. Yu H, Yang Q, Han M, et al. A case-control study on influencing factors of congenital heart disease in neonates in Shanghai, 2011. J Environ Occup Med. 2012;29:608-611.

27. Carey JC, Klebanoff MA, Hauth JC, et al. Metronidazole to prevent preterm delivery in pregnant women with asymptomatic bacterial vaginosis. $N$ Engl $J$ Med. 2000;342:534-540. doi:10.1056/ NEJM200002243420802

28. Greenland S, Pearl J, Robins JM. Causal diagrams for epidemiologic research. Epidemiology. 1999;10:37-48. doi:10.1097/00001648199901000-00008

29. Weng HY, Hsueh YH, Messam LL, Hertz-Picciotto I. Methods of covariate selection: directed acyclic graphs and the change-inestimate procedure. Am J Epidemiol. 2009;169:1182-1190. doi:10.1093/aje/kwp035

30. Textor J, Hardt J, Knuppel S. DAGitty: a graphical tool for analyzing causal diagrams. Epidemiology. 2011;22:745. doi:10.1097/ EDE.0b013e318225c2be

31. Zou L. The case-control study on risk factors of congenital heart disease, Wuyi. Chin J Birth Health Heredity. 2015;23:89-91.

32. Ou Y, Nie Z, Liu X, et al. Study on the differences of risk factors regarding congenital heart defects between floating population and permanent residents in Guangdong. Chin $J$ Epidemiol. 2013;34:701-705.

33. Chen L, Zheng X. Analysis on risk factors of congenital heart diseases. Pract Prev Med. 2012;19:32-34.

34. Zhao G, Jiang H, Pan R. Study on risk factors of congenital heart disease among fetuses and perinatal infants in Shenzhen. Matern Child Health Care China. 2011;26:5183-5185.

35. Chen G, Fang J, Wei S, Duan X, Wu Y, Liu X. Risk factors for congenital heart disease in Houjie Town of Dongguan Guangdong Province. South China J Cardiovasc Dis. 2010;16:376-381.

36. Liu H, Wang M, Dong Q, Chen S, Liu F, Zhang H. Study on risk factors of children with congenital heart disease in Hebei Province. Matern Child Health Care China. 2009;24:1496-1497.

37. Fung A, Manlhiot $\mathrm{C}$, Naik $\mathrm{S}$, et al. Impact of prenatal risk factors on congenital heart disease in the current era. J Am Heart Assoc. 2013;2: e64. doi:10.1161/JAHA.113.000064

38. Kourtis AP, Read JS, Jamieson DJ. Pregnancy and infection. $N$ Engl J Med. 2014;370:2211-2218. doi:10.1056/NEJMra1213566

39. Pazos M, Sperling RS, Moran TM, Kraus TA. The influence of pregnancy on systemic immunity. Immunol Res. 2012;54:254-261. doi:10.1007/s12026-012-8303-9

40. Robinson DP, Klein SL. Pregnancy and pregnancy-associated hormones alter immune responses and disease pathogenesis. Horm Behav. 2012;62:263-271. doi:10.1016/j.yhbeh.2012.02.023

41. Reef SE, Redd SB, Abernathy E, Zimmerman L, Icenogle JP. The epidemiological profile of rubella and congenital rubella syndrome in the United States, 1998-2004: the evidence for absence of endemic transmission. Clin Infect Dis. 2006;43(Suppl 3):S126-S132. doi: $10.1086 / 505944$
Clinical Epidemiology

\section{Publish your work in this journal}

Clinical Epidemiology is an international, peer-reviewed, open access, online journal focusing on disease and drug epidemiology, identification of risk factors and screening procedures to develop optimal preventative initiatives and programs. Specific topics include: diagnosis, prognosis, treatment, screening, prevention, risk factor modification, systematic reviews, risk \& safety of medical interventions, epidemiology \& biostatistical methods, and evaluation of guidelines, translational medicine, health policies \& economic evaluations. The manuscript management system is completely online and includes a very quick and fair peer-review system, which is all easy to use.

\section{Dovepress}

